\title{
Proteomic changes in maize as a response to heavy metal (lead) stress revealed by iTRAQ quantitative proteomics
}

\author{
G.K. Li ${ }^{1,3 *}$, J. Gao ${ }^{4 *}$, H. Peng ${ }^{1,2}$, Y.O. Shen ${ }^{1}$, H.P. Ding ${ }^{1}$, Z.M. Zhang ${ }^{1}$, G.T. Pan ${ }^{1}$ \\ and H.J. Lin ${ }^{1}$ \\ ${ }^{1}$ Key Laboratory of Biology and Genetic Improvement of Maize in Southwest Region, \\ Ministry of Agriculture, \\ Maize Research Institute of Sichuan Agricultural University, Wenjiang, Sichuan, China \\ ${ }^{2}$ Sichuan Tourism University, Chengdu, Sichuan, China \\ ${ }^{3}$ Guangdong Provincial Key Laboratory of Crops Genetics and Improvement, \\ Guangdong Academy of Agricultural Sciences, Crop Research Institute, Guangzhou, \\ China \\ ${ }^{4}$ Key Laboratory of Tumor Immunopathology, \\ Institute of Pathology and Southwest Cancer Center, Southwest Hospital, \\ Third Military Medical University, Ministry of Education, Chongqing, China \\ *These authors contributed equally to this study. \\ Corresponding authors: G.T. Pan / H.J. Lin \\ E-mail: pangt1956@aliyun.com / linhj521@gmail.com
}

Genet. Mol. Res. 15 (1): gmr.15017254

Received September 14, 2015

Accepted November 5, 2015

Published January 26, 2016

DOI http://dx.doi.org/10.4238/gmr.15017254

\begin{abstract}
Lead $(\mathrm{Pb})$, a heavy metal, has become a crucial pollutant in soil and water, causing not only permanent and irreversible health problems, but also substantial reduction in crop yields. In this study, we conducted proteome analysis of the roots of the non-hyperaccumulator inbred maize line 9782 at four developmental stages $(0,12,24$, and $48 \mathrm{~h})$ under $\mathrm{Pb}$ pollution using isobaric tags for relative and absolute quantification technology. A total of 252, 72 and 116 proteins were differentially expressed between M12 (after 12-h Pb treatment) and CK (water-mocked
\end{abstract}


treatment), M24 (after 24-h Pb treatment) and CK, and M48 (after 48-h $\mathrm{Pb}$ treatment) and $\mathrm{CK}$, respectively. In addition, 14 differentially expressed proteins were common within each comparison group. Moreover, Cluster of Orthologous Groups enrichment analysis revealed predominance of the proteins involved in posttranslational modification, protein turnover, and chaperones. Additionally, the changes in protein profiles showed a lower concordance with corresponding alterations in transcript levels, indicating important roles for transcriptional and posttranscriptional regulation in the response of maize roots to $\mathrm{Pb}$ pollution. Furthermore, enriched functional categories between the successive comparisons showed that the proteins in functional categories of stress, redox, signaling, and transport were highly up-regulated, while those in the functional categories of nucleotide metabolism, amino acid metabolism, RNA, and protein metabolism were down-regulated. This information will help in furthering our understanding of the detailed mechanisms of plant responses to heavy metal stress by combining protein and mRNA profiles.

Key words: Pb pollution; Isobaric tags for relative and absolute quantification; Differentially expressed proteins

\section{INTRODUCTION}

Plants are frequently subjected to various environmental stresses, such as cold, heat, drought, flooding, salt, light, and pollutants (including heavy metals) (Tester and Bacic, 2005), which have marked effects on plant vigor and crop yields. Recent studies have shown that environmental pollution due to heavy metals represents a serious threat to living organisms. Lead $(\mathrm{Pb}) \mathrm{has}$ become the most important metal pollutant of the environment because of widespread heavy metal pollution from agriculture, industry, and other human activities (Valko et al., 2005). Plants have developed various abilities to cope with and adapt to stress conditions by changing levels of protein, displaying a relative abundance of stress-responsive proteins in the proteome. To investigate the expression and modification of all proteins in an organism, high-throughput analyses for proteome studies have been developed, aided by advancements in mass spectrometry, genome sequencing, and bioinformatics (Miernyk and Hajduch, 2011).

Maize has a relatively high proportion of its biomass in its roots; therefore, maize could be an important model to study the phytoremediation of Pb-contaminated soil. Previous studies have indicated that a range of gene regulatory mechanisms control the accumulation capacity of $\mathrm{Pb}$ in plants, including transcription factors, transport proteins, and some critical genes, which were activated in response to both biotic and abiotic stresses and were involved in certain signal transduction and secondary metabolite pathways (Atkinson and Urwin, 2012; Thapa et al., 2012; Fan et al., 2013). In a previous study, we assayed $\mathrm{Pb}$ contents in the roots and aboveground parts of 19 inbred lines of maize seedlings. We selected the non-hyperaccumulator maize germplasm 9782 with $\mathrm{Pb}$ accumulation in roots, stems, and leaves under $\mathrm{Pb}$ conditions, and compared these individuals with the hyperaccumulator 178 (Zhang et al., 2012). In addition, transcriptomic changes during maize root development in response to $\mathrm{Pb}$ were investigated in the non-hyperaccumulator maize germplasm 9782 using RNA sequencing (Gao et al., 2015), as well as the dynamics of 
DNA methylation in maize germplasm 9782 roots under Pb stress (Ding et al., 2014). However, proteomic alteration profiling involved in various metabolic functions associated with $\mathrm{Pb}$ pollution is still uncertain.

In our research, considering that limited information is available with regard to the response of maize roots to $\mathrm{Pb}$ pollution, we generated a transcriptome, measuring the relative or absolute expression of protein during the response of maize roots to $\mathrm{Pb}$ stress. The recently developed isobaric tag for relative and absolute quantification (iTRAQ) system was used to accurately quantify differentially expressed proteins (DEPs), by measuring peak intensities of reporter ions in tandem mass spectrometry (MS/MS) spectra. DEPs were identified using ITRAQ and the expression patterns at the protein level correlated with transcript levels were investigated.

\section{MATERIAL AND METHODS}

\section{Plant materials and experimental design}

Seeds of maize (Zea mays) inbred line 9782 were sown on filter paper saturated with distilled water and incubated at $26^{\circ} \mathrm{C}$ in the dark. Seedlings with uniform growth were selected after three days, transplanted into an aerated complete nutrient solution (see Table S1), and grown in a growth chamber as follows: seedlings were maintained for three days with a 14-h light/10-h dark photoperiod at $26^{\circ} \mathrm{C}$ and a relative humidity of $70 \%$. After that, the maize seedlings were randomly divided into four groups. CK (water-mocked treatment)-grown (C1A) seedlings were grown only in half-strength Hoagland solution, while Pb1000-grown seedlings were grown in $\mathrm{CK}+\mathrm{Pb} 1000$ [1000 $\mathrm{mg} / \mathrm{L} \mathrm{Pb}\left(\mathrm{NO}_{3}\right)_{2}$ ] to achieve $\mathrm{Pb}$ stress for 12,24 , and $48 \mathrm{~h}$, respectively.

\section{Protein extraction}

Protein was extracted using the trichloroacetic acid (TCA)/acetone method (Sheoran et al., 2009) with modifications. Tuber cubes with $10 \%$ polyvinyl-polypyrrolidone were ground in liquid nitrogen and suspended in 5 volumes of chilled $\left(20^{\circ} \mathrm{C}\right)$ acetone containing $10 \% \mathrm{TCA}(\mathrm{w} / \mathrm{v})$. The homogenate was then precipitated for $2 \mathrm{~h}$ at $20^{\circ} \mathrm{C}$ and centrifuged at $20,000 \mathrm{~g}$ for $20 \mathrm{~min}$ at $4^{\circ} \mathrm{C}$. The supernatant was carefully removed, and the protein pellets were washed three times with chilled acetone. Washed protein pellets were air-dried and dissolved in $500 \mathrm{~mL} 0.5 \mathrm{M}$ triethylammonium bicarbonate (TEAB) for $15 \mathrm{~min}$ under $200 \mathrm{~W}$ sonication, and centrifuged at 30,000 $\mathrm{g}$ for $20 \mathrm{~min}$ at $4^{\circ} \mathrm{C}$. Then, the disulfide bonds were reduced in $10 \mathrm{mM}$ dithiothreitol at $56^{\circ} \mathrm{C}$ for $1 \mathrm{~h}$ and followed by alkylation in $55 \mathrm{mM}$ iodoacetamide for $45 \mathrm{~min}$. The supernatant was precipitated overnight at $20^{\circ} \mathrm{C}$ by the addition of 5 volumes of chilled acetone before centrifuging. The air-dried pellet was dissolved in $500 \mathrm{~mL} 0.5 \mathrm{M}$ TEAB for $15 \mathrm{~min}$ under $200 \mathrm{~W}$ sonication. The supernatant was collected as the soluble protein fraction after centrifugation at $30,000 \mathrm{~g}$ for $15 \mathrm{~min}$ at $4^{\circ} \mathrm{C}$. Protein concentration was estimated as a standard using the Bio-Rad protein assay kit (Bio-Rad, Hercules, CA, USA) with bovine serum albumin. The quality of each protein sample was evaluated using sodium dodecyl sulfate-polyacrylamide gel electrophoresis.

\section{iTRAQ labeling and mass spectrometry analysis}

iTRAQ labeling and mass spectrometry analysis were performed at the Beijing Genomics Institute (Shenzhen, China) according to the method described by Gan et al. (2007). From each 
sample, $100 \mu \mathrm{g}$ reduced and alkylated protein was digested using sequencing-grade trypsin (Promega Corporation, Beijing) and labeled using iTRAQ 8-plex kits (Applied Biosystems, USA) according to the manufacturer protocol. M12 samples were labeled with reagent 113 and 118 , M24 samples were labeled with reagent 114 and 119, and M48 samples were labeled with reagent 115 and 121. After labeling and quenching, the samples were combined and further fractionated by strong cation exchange (SCX) chromatography on an Ultramax SCX column using Shimadzu LC-20AB HPLC. The fractionated samples were analyzed by nano-LC-MS/MS. Peak lists were generated with the Proteome Discoverer 1.3 software.

\section{Proteomic data analysis}

Proteins were identified and quantified simultaneously using the Mascot software (version 2.3.02, Matrix Science), and then searched against the maize protein database with the following search parameters: trypsin was chosen as the enzyme with one missed cleavage allowed; fixed modifications of carbamidomethylation at Cys; variable modifications of oxidation at Met; peptide tolerance was set at $0.05 \mathrm{Da}$ and MS/MS tolerance at $0.1 \mathrm{Da}$; and monoisotopic mass was chosen. iTRAQ 8-plex was chosen for quantification during the search. Protein quantitation was performed at the peptide level by following the procedures described in http://www.matrixscience.com/help/ quant_statistics_help.html. Proteins with changes of 1.5-fold or greater between successive comparisons and a $\mathrm{P}$ value of statistical evaluation less than 0.05 were determined as differentially expressed proteins. The MapMan 3.6.0RC1 tool (http://mapman.gabipd.org/web/guest) was used to display expression profiles at the pathway level. Expression values were calculated based on a log2 scale and used to visualize the metabolic processes.

\section{Comparison of protein and mRNA expression profiles}

To compare changes in protein abundance with alterations in transcript levels, we conducted RNA sequencing experiments using the same materials as described in protein extraction. RNA extraction (Cat. No. 15596-026, Invitrogen) and RNA sequencing library construction (Illumina protocol) were performed following manufacturer instructions. In this study, the quantified proteins were quantitatively compared and clustered into four groups based on mRNA and protein levels. These groups were i) mRNA and protein levels having the same trends, ii) mRNA and protein levels having opposite change trends, iii) protein levels changing significantly while the mRNA was unchanged, and iv) mRNA levels changing significantly while the protein remains unchanged. Pearson correlation tests were conducted using expression ratios of M12 vs CK, M24 vs CK, and M48 vs CK, respectively.

\section{RESULTS}

\section{Overview of quantitative proteome analysis}

The proteome of maize roots responsive to $\mathrm{Pb}$ pollution were quantitatively cataloged using ITRAQ technology. Briefly, proteins were extracted, digested, and iTRAQ-labeled in solution. Of those, unlabeled peptides were excluded from the data sets; iTRAQ-labeled peptides were analyzed using liquid chromatography combined with tandem mass spectroscopy. Of the 352,944 
mass spectra obtained that matched to special peptides, low-scoring spectra were eliminated by data filtering, and 24,151 high-scoring unique spectra were identified. Of those, 6029 unique peptides were identified from 7442 peptides, corresponding to 2440 proteins in the four samples (Figure 1A). In terms of protein mass distribution, good coverage (averages of $10-16 \%$ of the total proteins in each protein mass group) was obtained for a wide protein mass (PS range for proteins larger than $10 \mathrm{kDa}$ and less than $70 \mathrm{kDa}$ ) (Figure 1B). In addition, peptide coverage in the proteins was identified; more than $48 \%$ of the proteins had more than $5 \%$ of sequence coverage (Figure 1C). Moreover, most of the proteins were identified with good distribution of sequence coverage; $70 \%$ of the proteins had over $10 \%$ of the sequence coverage and $56 \%$ were with $20 \%$ of the sequence coverage (Figure 1D). To quantify their abundance, a subset protein was identified by at least two peptides (Table S1). Of the proteins detected, 2112, 2091, 2236, 2238, 2217 , and 2076 were identified from samples of M12_re1, M12_re1, M24_re1, M24_re2, M48_re1, and M48_re2, respectively. Comparisons between various biological replicates are shown in Figure 2 to demonstrate analytical reproducibility. We labeled and mixed each pair of Pb-treated sample and its control for proteomic analysis; the results showed that $96.09,98.55$, and $96.34 \%$ of the proteins were detected in all six biological samples for M12, M24, and M48, respectively (Figure 2). In addition, two biological replicates of $\mathrm{Pb} 1000$-treated samples were labeled and mixed for proteomic analysis, and the percentage of proteins revealed, suggesting that biological noise was reasonably low and the analysis is reliable for protein identification (Figure 3).
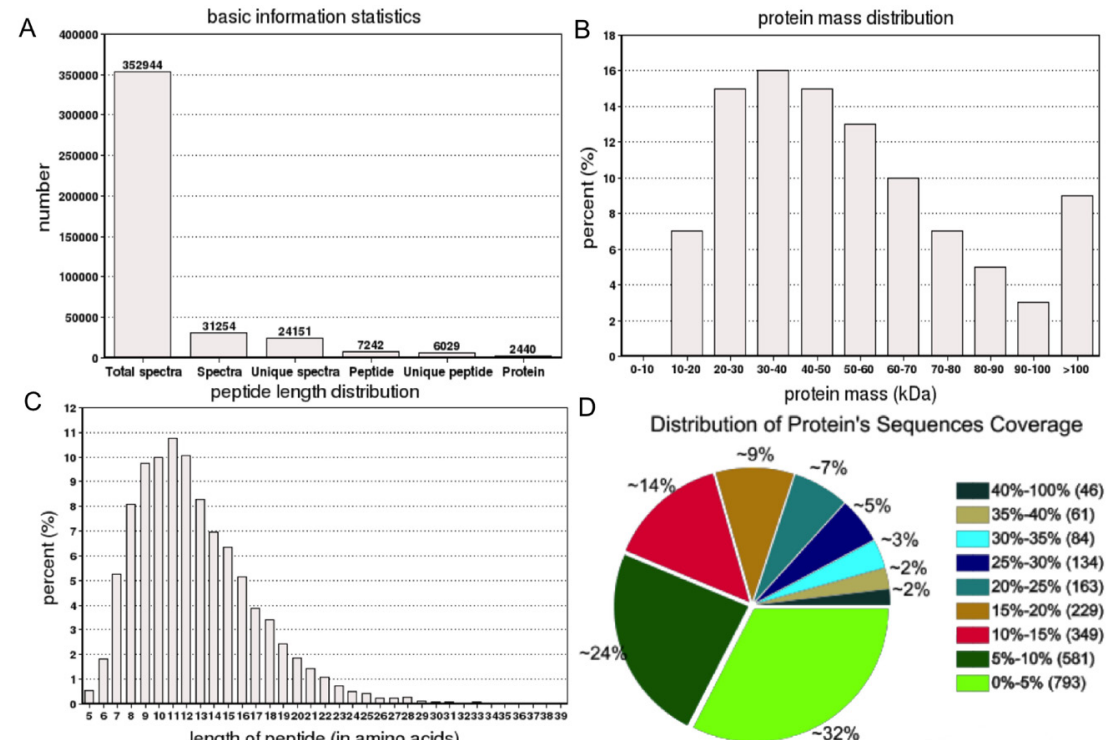

Figure 1. Analysis of maize roots responsive to $\mathrm{Pb}$ pollution proteome profile by iTRAQ. Total spectra are the secondary mass spectrums, and spectra are the secondary mass spectrums after quality control. Unique Peptide is the identified peptides that belong only to a group of proteins, and protein is identified by the Mascot 2.3.02 software. A. High scoring unique spectra matched to special peptides were obtained, unique peptides were identified from peptides corresponding to proteins in the four samples were identified. B. Obtaining of good coverage of protein mass distribution. $\mathbf{C}$. Identification of length peptide coverage in the proteins. D. Identification of proteins with good distribution of peptide coverage. Total Spectra is the total for the secondary spectra, Spectra is the number of matched spectra, Unique Spectra is the the number of spectrum to matched unique peptides, Peptide is the number of peptides by identify, Unique Peptide is the sequence number of the specific peptide by identify is segments, Protein is the the amount of protein by identify. 


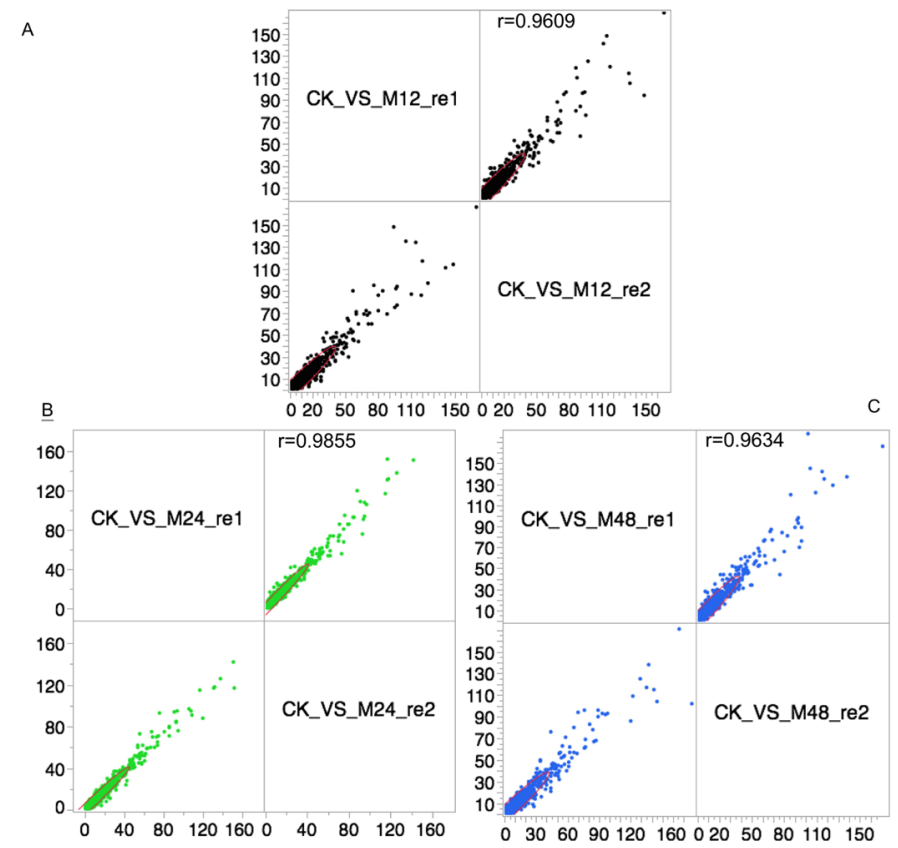

Figure 2. Comparisons between various biological replicates to demonstrate the analytical reproducibility, each pair of $\mathrm{Pb}$-treated samples and its control for proteomic analysis were labeled in all six biological samples for M12, M24 and M48, respectively.
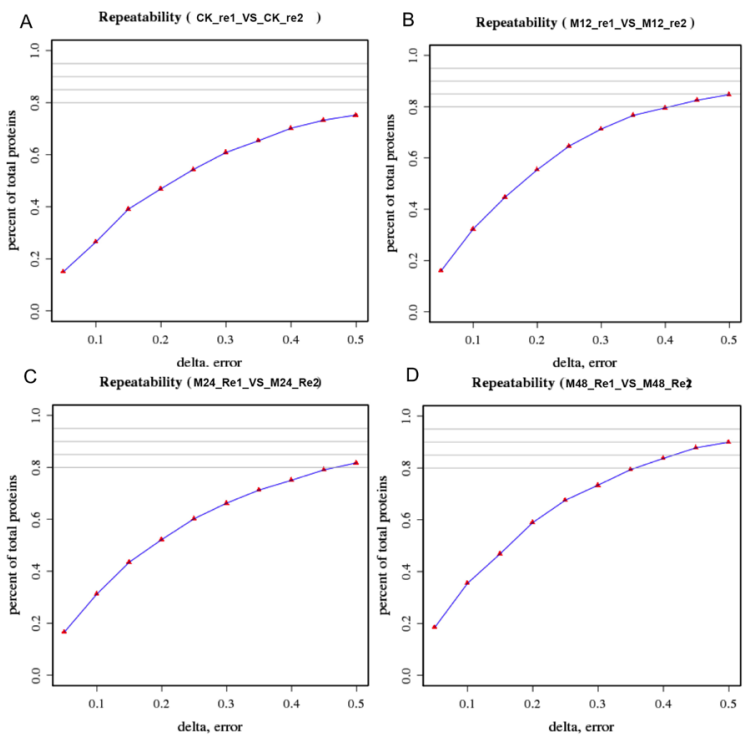

Figure 3. Different proteins identified by two biological replicates. To further elucidate the global function of proteins responsive to $\mathrm{Pb}$ pollution, the detected proteins were categorized into different functional groups based on the Cluster of Orthologous Groups (COG) database. Expect for general function prediction only, the percentages of proteins involved in posttranslational modification, protein turnover, and chaperones were dominant, indicating that posttranscriptional regulation plays an vital roles in maize roots responsive to $\mathrm{Pb}$ pollution (Figure 4). 


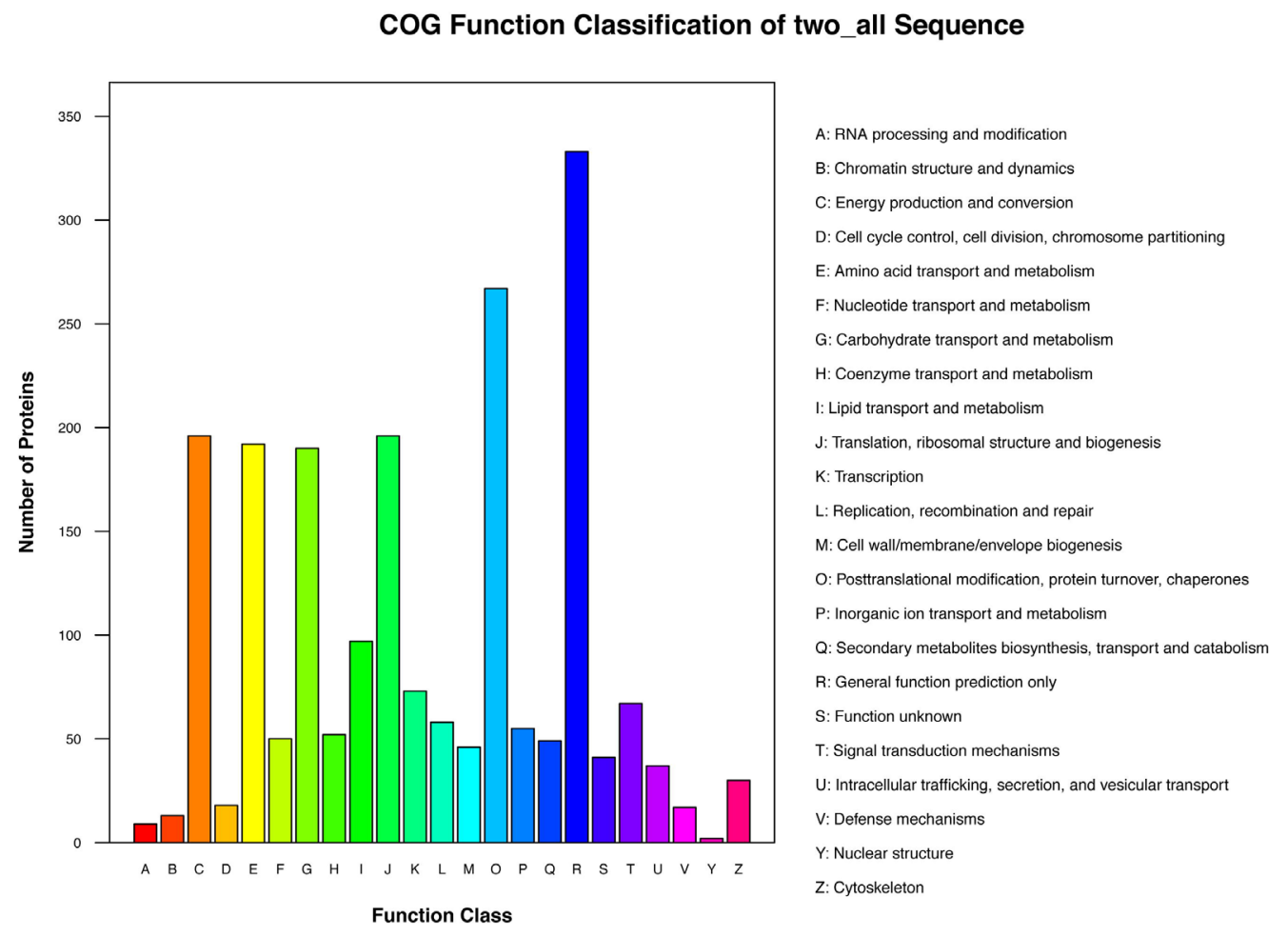

Figure 4. Cluster of Orthologus Groups (COG) analysis of differentially expressed proteins in maize roots responsive to $\mathrm{Pb}$ pollution. Shown above is the classification of these proteins in different categories based on biological process.

\section{Functional annotation analysis of differences in proteome}

To further identify the DEPs in maize roots under $\mathrm{Pb}$ stress, proteins with $>1.5$-fold difference and $P$ value $<0.05$ were regarded as being differentially expressed (Gan et al., 2007). $\mathrm{DEP}$ levels of the developmental stages after $\mathrm{Pb}$ pollution were compared with those of the mocktreatment control (M12 vs CK, M24 vs CK and M48 vs CK). Of these, 252 proteins were screened as differentially expressed between M12 and CK, including 115 proteins that were up-regulated and 137 that were down-regulated; 72 proteins experienced significant up- (35) or down-regulation (37) between M24 and CK and 116 proteins experienced significant up- (57) or down-regulation (59) between M48 and CK (Table 1). In addition, 14 DEPs were found to be common between M12 and CK, M24 and CK, and M48 and CK. Cluster analysis showed that 4 DEPs were increased and 10 DEPs were induced after Pb1000 treatment. However, most of them were significantly increasing during the maize roots' response to $\mathrm{Pb}$ treatment (Figure 5). Interestingly, based on the differentially expressed genes identified by RNAseq in our study, most DEPs also were identified in the $\mathrm{M} 12$ vs $\mathrm{CK}$ comparison, suggesting that $\mathrm{M} 12$ represents the most active period of the response of maize roots to $\mathrm{Pb}$ stress. 
Table 1. DEPs between M48 and CK.

\begin{tabular}{|c|c|c|c|c|c|}
\hline Accession No. & Cov & Unique Spectrum & Unique Peptide & Log2 (Treat/CK) & Description \\
\hline GRMZM2G024354_P01 & 19.6 & 17 & 1 & 2.43 & $60 S$ ribosomal protein $\mathrm{L} 15$ \\
\hline GRMZM2G358059_P01 & 24.9 & 3 & 1 & 2.41 & Calreticulin2 \\
\hline GRMZM2G015361_P01 & 27.6 & 23 & 5 & 2.11 & GTP binding \\
\hline GRMZM2G039639_P01 & 4.8 & 7 & 1 & 1.79 & Protein P21 \\
\hline GRMZM2G002825_P01 & 15.8 & 17 & 2 & 1.59 & Actin-depolymerizing factor 3 \\
\hline GRMZM2G014240_P01 & 12.3 & 7 & 2 & 1.38 & Inorganic diphosphatase activity \\
\hline AC233895.1_FGP001 & 17.8 & 28 & 7 & 1.08 & Proliferation-associated protein $2 \mathrm{G} 4$ \\
\hline GRMZM2G052562_P01 & 2.5 & 6 & 1 & 0.93 & Zea nodulation homolog1 \\
\hline GRMZM2G036099_P03 & 13.6 & 8 & 2 & 0.91 & Calcium ion binding \\
\hline GRMZM2G162426_P01 & 3.4 & 6 & 3 & 0.89 & Catalytic activity \\
\hline GRMZM5G877500_P01 & 5.1 & 5 & 2 & 0.87 & Catalytic activity \\
\hline GRMZM2G033208_P01 & 14.4 & 38 & 7 & 0.80 & Catalytic activity \\
\hline GRMZM2G015361_P01 & 27.6 & 23 & 5 & 0.70 & GTP binding \\
\hline GRMZM2G113696_P01 & 18.8 & 21 & 3 & 0.69 & Translation initiation factor $5 \mathrm{~A}$ \\
\hline GRMZM2G085967_P01 & 29.0 & 65 & 8 & 0.69 & Peroxidase 39 \\
\hline GRMZM2G358059_P01 & 24.9 & 3 & 1 & 0.66 & Calreticulin2 \\
\hline GRMZM2G008247_P01 & 20.2 & 12 & 1 & 0.65 & Beta-D-glucosidase precursor \\
\hline GRMZM2G058675_P02 & 29.3 & 42 & 9 & 0.65 & Restorer of fertility2 \\
\hline GRMZM2G109677_P03 & 15.2 & 37 & 7 & 0.62 & Structural constituent of ribosome \\
\hline GRMZM2G109677_P03 & 15.2 & 37 & 7 & 0.62 & Structural constituent of ribosome \\
\hline GRMZM2G178693_P01 & 14.4 & 15 & 3 & 0.60 & Plasma membrane intrinsic protein \\
\hline GRMZM2G154169_P01 & 14.5 & 9 & 2 & -0.62 & GRF-interacting factor 2-like \\
\hline GRMZM2G107073_P01 & 11.7 & 23 & 4 & -0.63 & Glycoside hydrolase, family 28 \\
\hline GRMZM2G000326_P01 & 38.6 & 18 & 2 & -0.63 & Subtilisin-chymotrypsin inhibitor $\mathrm{Cl}-1 \mathrm{~B}$ \\
\hline GRMZM2G305046_P01 & 28.9 & 12 & 1 & -0.64 & Histone2A1 \\
\hline GRMZM2G038636_P01 & 6.4 & 12 & 2 & -0.68 & Cysteine protease \\
\hline GRMZM2G050514_P01 & 33.1 & 10 & 4 & -0.68 & Glutamine synthetase \\
\hline AC197758.3_FGP004 & 15.9 & 21 & 3 & -0.70 & Peroxidase 52 \\
\hline GRMZM2G145440_P01 & 7.5 & 3 & 1 & -0.73 & Receptor kinase 1 \\
\hline GRMZM2G117989_P01 & 5.1 & 9 & 1 & -0.81 & Defense response to bacterium \\
\hline GRMZM2G146246_P02 & 36.2 & 21 & 6 & -0.82 & Glutathione S-transferase4 \\
\hline GRMZM2G003306_P01 & 29.5 & 10 & 1 & -0.83 & Nucleosome \\
\hline GRMZM2G057608_P01 & 28.7 & 16 & 3 & -0.90 & $40 \mathrm{~S}$ ribosomal protein $\mathrm{S} 25-1$ \\
\hline GRMZM2G156632_P01 & 9.8 & 12 & 1 & -1.02 & WIP1 \\
\hline GRMZM2G039639_P01 & 4.8 & 7 & 1 & -1.03 & Protein P21 \\
\hline GRMZM2G374971_P01 & 16.7 & 14 & 3 & -1.13 & Stress-induced protein 1 \\
\hline GRMZM2G005633_P02 & 21 & 8 & 3 & -1.15 & Chitinase activity \\
\hline GRMZM2G304442_P01 & 20.1 & 15 & 3 & -1.35 & Extracellular region \\
\hline GRMZM2G075624_P01 & 24.0 & 16 & 2 & -1.35 & Translationally controlled tumor protein-like Protein \\
\hline GRMZM2G078314_P01 & 16.9 & 4 & 1 & -1.36 & Nucleosome \\
\hline GRMZM2G031545_P01 & 23.6 & 15 & 4 & -1.44 & Elongation factor 1 -delta 1 \\
\hline GRMZM2G057608_P01 & 28.7 & 16 & 3 & -1.49 & 40S ribosomal protein S25-1 \\
\hline GRMZM2G051879_P03 & 28.3 & 2 & 2 & -1.91 & Nucleosome \\
\hline GRMZM2G158568_P01 & 11.4 & 7 & 2 & -1.97 & $60 S$ ribosomal protein $\mathrm{L} 31$ \\
\hline GRMZM2G117989_P01 & 5.1 & 9 & 1 & -2.77 & Defense response to bacterium \\
\hline GRMZM2G083016_P01 & 15.7 & 6 & 2 & -13.35 & Phosphoglycerate kinase activity \\
\hline
\end{tabular}

Accession represent the protein code; coverage values represent the coverage (\%) of each protein assigned by a peptides; unique peptide represent the unique peptide align to the protein; CK vs M48 represent the protein abundance at $\mathrm{CK}$ is compared to that at M48.

To further study the function of proteins related to $\mathrm{Pb}$ stress responsiveness, DEPs were also clustered according to expression changes during the developmental stages after $\mathrm{Pb}$ pollution-most proteins between M12 vs CK, M24 vs CK, and M48 vs CK demonstrated forward expression patterns using the MapMan program. Additionally, enriched functional categories between the successive comparisons showed that the proteins in functional categories of stress, redox, signaling, and transport were highly up-regulated, while proteins in functional categories of nucleotide metabolism, amino acid metabolism, RNA, and protein metabolism were downregulated (Figure 6). 

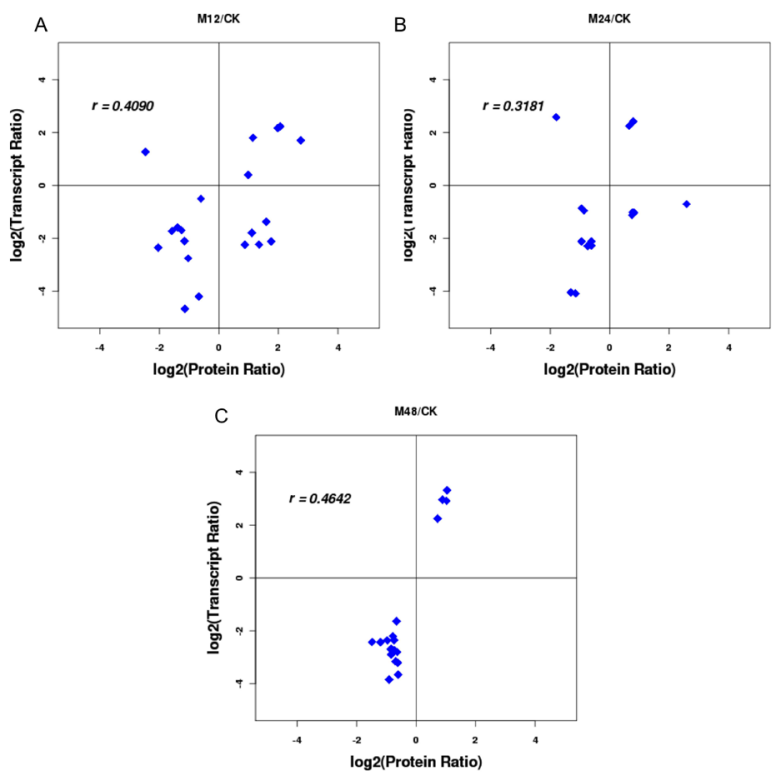

Figure 5. Common DEPs identified shared between each group, such as M12 vs CK, M24 vs CK and M48 vs CK. Cluster analysis showed increased and decreased DEPs induced by $\mathrm{Pb}$ treatment.

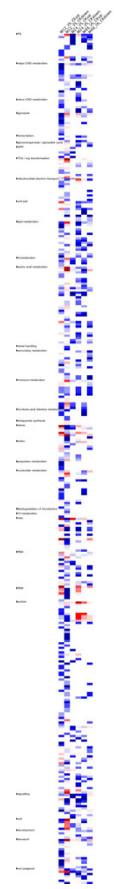

Figure 6. Differentially expressed proteins (DEPs) corresponded to expression changes during the developmental stages after $\mathrm{Pb}$ pollution. Clustered and enriched functional categories between the successive comparisons were conducted using MapMan program. 
These results suggested that the stress and redox-related metabolism was mainly activated during the developmental stages after $\mathrm{Pb}$ pollution, and the metabolites, along with related signaling pathway and transport proteins, also increased to respond to $\mathrm{Pb} 1000$ treatment (Table S2).

\section{Comparison of transcriptome and proteome expression}

RNA sequencing provided the following numbers of high-quality clean reads from the respective stages: 19,562,150 from CK, 19,171,257 from M12, 19,248,546 from M24, and $17,813,916$ from M48. These corresponded to 17,707, 17,440, 16,998, and 16,586 genes identified in maize roots at the four developmental stages $(0,12,24$, and $48 \mathrm{~h})$, respectively. Also, 2825, 2626, 2161, and 2260 stage-specific genes at successive time points were screened, based on the absolute fold change value of $\log 2$ ratio $>1$ with $\mathrm{P}<0.001$ and FDR $<0.001$ (data not shown).

To compare changes in protein abundance with alterations in transcript levels in maize roots responsive to $\mathrm{Pb} 1000$ treatment, the correlation between protein and mRNA expression profiles were further comprehensively investigated by comparing M12 vs CK, M24 vs CK, and M48 vs CK. Of 252 proteins that changed significantly in terms of abundance between M12 vs CK, only 41 of their corresponding transcripts were found to be differentially expressed. For M24 vs CK and M48 vs CK, of 72 and 116 proteins corresponding to 19 and 37 transcripts, respectively, some DEPs have the same or opposite change trends with their association mRNA in transcriptome level, some DEPs were significantly changed in proteasome level but the cognate mRNAs were not significantly changed. (Table 2). Next, the correlation between differentially expressed proteins and mRNAs was comprehensively investigated in the comparison of M12 vs CK, M24 vs CK, and M48 vs CK. Concordance tests revealed a positive correlation of $r$ of $0.409,0.318$, and 0.4642 between protein and mRNAs for the significant change (Figure 7). This indicated that a change in transcript abundance may or may not translate into changes in protein level. Posttranscriptional regulatory processes such as protein-protein interactions, redox systems, processes of reversible phosphorylation, and some mediatory molecules may affect the efficiency of translation.

\section{DISCUSSION}

\section{DEPs between Pb1000-grown and control groups}

In the current study, the effects of heavy metal lead $(\mathrm{Pb})$ on the proteome was investigated using quantitative proteomics to uncover $\mathrm{Pb}$-regulated protein regulatory mechanisms in maize roots. However, previous studies have shown that transcription and translation do not always correlate well with each other. Therefore, our proteomic analysis may reveal novel players involved in heavy metal regulatory pathways. The ITRAQ method was used to identify putative DEPs potentially responsive to heavy metal pollution, and 14 DEPs were identified as being common within each comparison group (M12 vs CK, M24 vs CK, M48 vs CK). Of these 14 common DEPs in all examined time points, two plasma membrane intrinsic proteins (GRMZM2G178693_P01 and GRMZM2G154628_P01) were identified among the proteins that were up-regulated in M24 $\mathrm{Pb}$-pollution conditions and then down-regulated in M48. Plasma membrane intrinsic proteins (PIPs), a subfamily of aquaporins, can control the translocation of water across the membrane. Previous studies have reported that, in various plant species, gene expression and function of 
Proteomic changes in response to lead stress

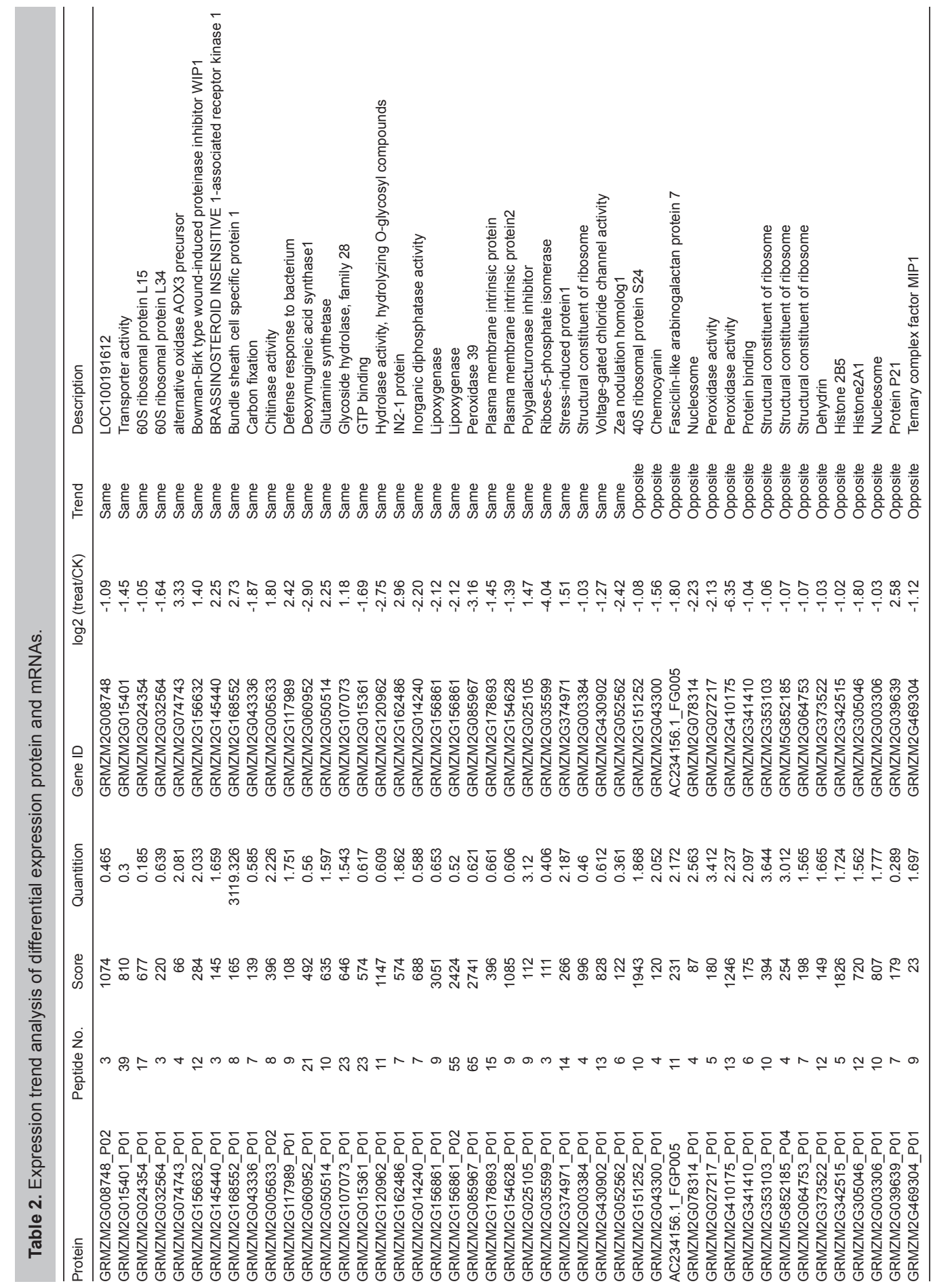



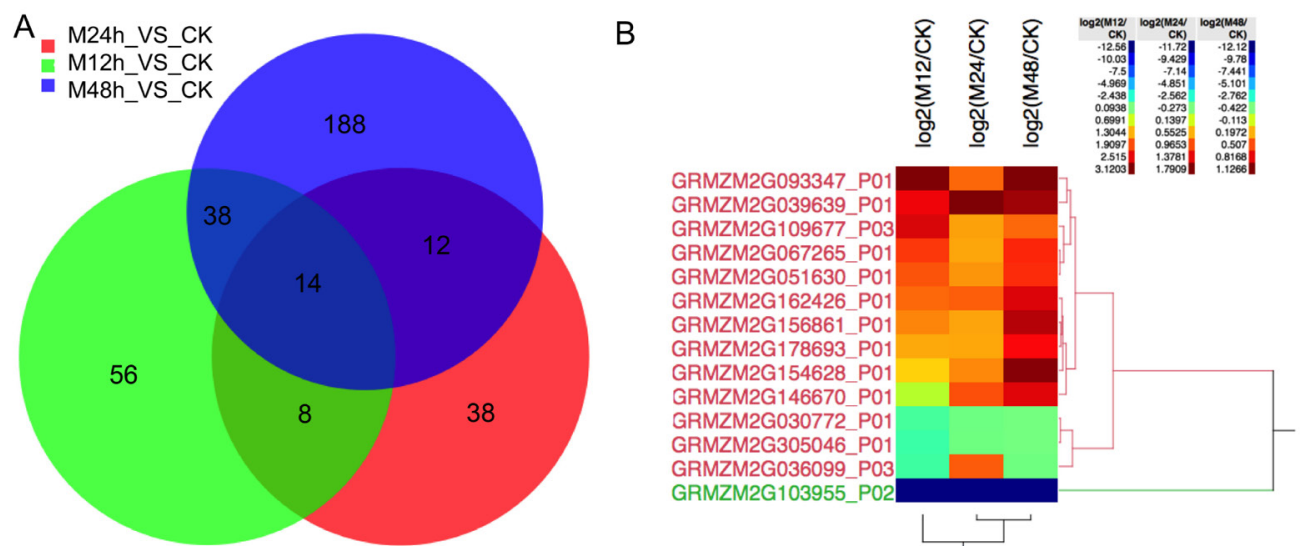

Figure 7. Correlation analysis between differentially expressed proteins and mRNA was comprehensively investigated in the comparison of each group, such as M12 vs CK, M24 vs CK and M48 vs CK. Concordance tests between protein and mRNAs for the significantly change were also conducted.

PIPs can be modulated by heavy metals such as cadmium, copper, and mercury (Guo et al., 2006). Expression of PsPIP2-1 showed an increasing trend after $\mathrm{HgCl}_{2}$ [mercury (II) chloride] treatment in Pisum sativum (Beaudette et al., 2007), whereas copper stress genes encoding plasmalemma PIP were down-regulated under copper application in Populus deltoides roots (Guerra et al., 2009). Similarly, 15 tags annotated to PIPs were significantly down-regulated, while two tags annotated to Arabidopsis PIPs were up-regulated in response to cadmium stress in Solanum torvum. Moreover, coexpression of nonfunctional ZmPIP1;1 and ZmPIP1;2 isoforms with functional ZmPIP2 protein could enhance their aquaporin activity significantly in maize (Fetter et al., 2004). Further study suggested that PIP1 trafficking would be required for the plasma membrane by PIP1-PIP2 interaction, thereby modulating plasma membrane permeability (Zelazny et al., 2007). We demonstrated that PIPs might facilitate the transport of molecules by modulating plasma membrane permeability in response to $\mathrm{Pb}$ pollution.

In addition, most of these down-regulated proteins are metal-ion binding, involved in heavy metal detoxification systems by the activation of metal ion transport pathways; for example, manganese ion binding (GRMZM2G030772_P01) and calcium ion binding (GRMZM2G036099 P03). Transporter proteins mediate metal uptake in root cells and metal transfer between cells and organs. Metal transporters are also involved in metal detoxification by mediating the transport of metals from the cytosol to the vacuolar compartment (Salt and Wagner, 1993; Salt and Rauser, 1995; Rea, 1999).

Moreover, numerous studies have demonstrated that chromatin regulation is involved in the expression of stress-associated genes, including protein P21 (GRMZM2G039639_P01) and histone2A1 (GRMZM2G305046_P01), as well as the structural constituent of ribosome protein (GRMZM2G109677_P03).

\section{$\mathrm{Pb}$-stress alters the expression of proteins in lipid metabolism and redox pathways}

MapMan analysis was used to evaluate whether significant DEPs with great alteration due 
to $\mathrm{Pb}$ stress were clustered in specific metabolic pathways. As expected, there was an increase in abundance of many proteins involved in amino acid metabolism as well as lipid and hormone metabolism in response to $\mathrm{Pb}$ stress. Two proteins with increased expression were found to be involved in lipid synthesis. Lipid metabolism is affected by heavy metal-induced oxidative stress, in varying degrees depending on concentration and tissue types (Gao et al., 2010).

In addition, some DEPs were identified as regulators for redox pathways. In plants, cellular redox homeostasis can sense and transfer reducing equivalents to numerous target proteins, which is maintained by $N A D(P) H$-dependent thioredoxin and glutaredoxin systems and involved in various adverse environments (Meyer et al., 2012). In addition, several studies have revealed that glutathione (GSH) acts as an important protection system against metal stress in plants. In Arabidopsis thaliana, Vanhoudt et al. (2010) demonstrated that the redox balance of GSH plays a vital role in protecting against uranium and cadmium stress. In Vicia faba, GSH content was increased by mercury stress level and related to the elimination of $\mathrm{H}_{2} \mathrm{O}_{2}$ via the ascorbate $\mathrm{GSH}$ cycle (Wang et al., 2010). Kalinowska and Pawlik-Skowrońska (2010) also reported that maintenance of a higher level of GSH was responsible for a higher resistance to copper concomitant with phytochelatin production.

In summary, DEPs associated with Pb stress were identified in our iTRAQ studies and most of them were found to be involved in lipid metabolism and redox pathways. Further characterization of these $\mathrm{Pb}$-regulated proteins may provide new insights into the molecular mechanism of action of lead pollution in plants.

\section{Conflicts of interest}

The authors declare no conflict of interest.

\section{ACKNOWLEDGMENTS}

Research supported by grants from the National Science Foundation of China (\#31171567), the Research Project on Rice Functional Genes related to the Maize Large-scale Gene Discovery and Functional Genomics Resource, Technology, Information Platform Construction Program "863" of China (\#SS2012AA100107 and \#2012AA10A300), the Science and Information Technology Bureau of Guangzhou (\#2013J2200083), the Guangdong Province and Ministry of Education Production-Study-Research Program of China (\#2012B091100467), the Foundation of the President of the Guangdong Academy of Agricultural Sciences (201407), and the Guangdong Province Science and Technology Program (\#2013B020301015).

\section{REFERENCES}

Atkinson NJ and Urwin PE (2012). The interaction of plant biotic and abiotic stresses: from genes to the field. J. Exp. Bot. 63: 3523-3543. http://dx.doi.org/10.1093/jxb/ers100

Beaudette PC, Chlup M, Yee J and Emery RJ (2007). Relationships of root conductivity and aquaporin gene expression in Pisum sativum: diurnal patterns and the response to $\mathrm{HgCl} 2$ and ABA. J. Exp. Bot. 58: 1291-1300. http://dx.doi. org/10.1093/jxb/erl289

Ding H, Gao J, Qin C, Ma H, et al. (2014). The dynamics of DNA methylation in maize roots under Pb stress. Int. J. Mol. Sci. 15: 23537-23554. http://dx.doi.org/10.3390/ijms151223537

Fan XD, Wang JQ, Yang N, Dong YY, et al. (2013). Gene expression profiling of soybean leaves and roots under salt, salinealkali and drought stress by high-throughput Illumina sequencing. Gene 512: 392-402. http://dx.doi.org/10.1016/j. 
gene. 2012.09.100

Fetter K, Van Wilder V, Moshelion M and Chaumont F (2004). Interactions between plasma membrane aquaporins modulate their water channel activity. Plant Cell 16: 215-228. http://dx.doi.org/10.1105/tpc.017194

Gan CS, Chong PK, Pham TK and Wright PC (2007). Technical, experimental, and biological variations in isobaric tags for relative and absolute quantitation (iTRAQ). J. Proteome Res. 6: 821-827. http://dx.doi.org/10.1021/pr060474i

Gao J, Zhang Y, Lu C, Peng H, et al. (2015). The development dynamics of the maize root transcriptome responsive to heavy metal $\mathrm{Pb}$ pollution. Biochem. Biophys. Res. Commun. 458: 287-293. http://dx.doi.org/10.1016/j.bbrc.2015.01.101

Gao S, Ou-yang C, Tang L, Zhu JQ, et al. (2010). Growth and antioxidant responses in Jatropha curcas seedling exposed to mercury toxicity. J. Hazard. Mater. 182: 591-597. http://dx.doi.org/10.1016/j.jhazmat.2010.06.073

Guerra F, Duplessis S, Kohler A, Martin F, et al. (2009). Gene expression analysis of Populus deltoides roots subjected to copper stress. Environ. Exp. Bot. 67: 335-344. http://dx.doi.org/10.1016/j.envexpbot.2009.08.004

Guo L, Wang ZY, Lin H, Cui WE, et al. (2006). Expression and functional analysis of the rice plasma-membrane intrinsic protein gene family. Cell Res. 16: 277-286. http://dx.doi.org/10.1038/sj.cr.7310035

Kalinowska R and Pawlik-Skowrońska B (2010). Response of two terrestrial green microalgae (Chlorophyta, Trebouxiophyceae) isolated from Cu-rich and unpolluted soils to copper stress. Environ. Pollut. 158: 2778-2785. http://dx.doi.org/10.1016/j. envpol.2010.03.003

Meyer Y, Belin C, Delorme-Hinoux V, Reichheld JP, et al. (2012). Thioredoxin and glutaredoxin systems in plants: molecular mechanisms, crosstalks, and functional significance. Antioxid. Redox Signal. 17: 1124-1160. http://dx.doi.org/10.1089/ ars.2011.4327

Miernyk JA and Hajduch M (2011). Seed proteomics. J. Proteomics 74: 389-400. http://dx.doi.org/10.1016/j.jprot.2010.12.004

Rea PA (1999). MRP subfamily ABC transporters from plants and yeast. J. Exp. Bot. 50: 895-913. http://dx.doi.org/10.1093/ ¡xb/50.Special Issue. 895

Salt DE and Wagner GJ (1993). Cadmium transport across tonoplast of vesicles from oat roots. Evidence for a $\mathrm{Cd} 2+/ \mathrm{H}+$ antiport activity. J. Biol. Chem. 268: 12297-12302.

Salt DE and Rauser WE (1995). MgATP-dependent transport of phytochelatins across the tonoplast of oat roots. Plant Physiol. 107: 1293-1301.

Sheoran IS, Ross AR, Olson DJ and Sawhney VK (2009). Compatibility of plant protein extraction methods with mass spectrometry for proteome analysis. Plant Sci. 176: 99-104. http://dx.doi.org/10.1016/i.plantsci.2008.09.015

Tester M and Bacic A (2005). Abiotic stress tolerance in grasses. From model plants to crop plants. Plant Physiol. 137: 791793. http://dx.doi.org/10.1104/pp.104.900138

Thapa G, Sadhukhan A, Panda SK and Sahoo L (2012). Molecular mechanistic model of plant heavy metal tolerance. Biometals 25: 489-505. http://dx.doi.org/10.1007/s10534-012-9541-y

Vanhoudt N, Vandenhove H, Horemans N, Wannijn J, et al. (2010). The combined effect of uranium and gamma radiation on biological responses and oxidative stress induced in Arabidopsis thaliana. J. Environ. Radioact. 101: 923-930. http:// dx.doi.org/10.1016/j.jenvrad.2010.06.008

Valko M, Morris H and Cronin MT (2005). Metals, toxicity and oxidative stress. Curr. Med. Chem. 12: 1161-1208. http://dx.doi. org/10.2174/0929867053764635

Wang CR, Tian Y, Wang XR, Yu HX, etal. (2010). Hormesis effects and implicative application in assessment of lead-contaminated soils in roots of Vicia faba seedlings. Chemosphere 80: 965-971. http://dx.doi.org/10.1016/j.chemosphere.2010.05.049

Zelazny E, Borst JW, Muylaert M, Batoko H, et al. (2007). FRET imaging in living maize cells reveals that plasma membrane aquaporins interact to regulate their subcellular localization. Proc. Natl. Acad. Sci. USA 104: 12359-12364. http://dx.doi. org/10.1073/pnas.0701180104

Zhang Z, Jin F, Wang C, Luo J, et al. (2012). Difference between Pb and Cd accumulation in 19 elite maize inbred lines and application prospects. J. Biomed. Biotechnol. 2012: 271485 http://dx.doi.org/10.1155/2012/271485.

\section{Supplementary material}

Table S1. Elementary proteomics data of maize roots repsonsive to Lead $(\mathrm{Pb})$ revealed by iTRAQ.

Table S2. Differentially expressed proteins involved in different metabolic pathways.

www.geneticsmr.com/year2016/vol15-1/pdf/gmr7254_supplementary.pdf 